\title{
Editorial: the role and influence of key stakeholders in the learning technology community
}

In this issue of ALT-J we have six articles that address three broad topics of video, costbenefit analysis and the emergent learning technology community. The first two articles present case studies on the use of video in teaching and learning. Shephard et al. describe their experiences of re-purposing a back 'care video' for video streaming, and use these experiences to highlight key challenges that others may face when attempting to re-purpose a video. Blake and Scanlon use their experiences of analysing video recordings of students, who were using computers to support their collaboration in solving statistical problems, to argue that such video analysis provides useful rich data with which to interpret and understand students' experiences.

The third and fourth articles address the challenging issue of assessing the costs and benefits of both specific and general learning technologies. Loewenberger and Bull present the results of a survey that attempted to gather information about the costs and benefits of computer-based assessment; while Nicol and Coen describe and illustrate a model that has been developed to enable evaluations of the costs and benefits of ICT.

The final two articles debate issues raised by an article by Lisewski and Joyce, entitled 'Examining the five-stage e-moderating model: designed and emergent practice in the learning technology profession', published in issue 11.1 of ALT-J. Tompsett and Alsop present a commentary on this article and offer a reinterpretation of the research by taking what they call 'a stricter analysis' of the events described by Lisewski and Joyce. In a response to this reinterpretation Joyce and Lisewski argue that the approach taken by Tompsett and Alsop is too narrow and runs the risk of not taking into account the whole context in which learning technology as a profession is emerging.

When I read the six articles, a quote by Frank DeRuyter, an assistive technology researcher in America, came to my mind. In discussing methods for evaluating the success of assistive technologies he wrote:

While it is reasonable to assume that each stakeholder desires a successful outcome, it is 
unreasonable to assume that each stakeholder desires the same outcome. (DeRuyter, 1995)

Picking up on this idea, it seems to me that one theme that draws all the articles in this issue of $A L T-J$ together is that of 'stakeholders' and the different views that different stakeholders may have on the costs, benefits, successes or failures of learning technologies. In the article by Shephard et al. article the stakeholders can be seen to be the funders, the lecturers, the media producer, the learning technology coordinator as well as the actors and patients in the video. The different views and interests of all these stakeholders presented the authors with a number of challenges when trying to re-purpose a video for video streaming. For example, the ownership of copyright, creative and moral rights by various stakeholders in the video meant that the video had to be cut or re-edited in places and that restrictions were placed on where the video could be accessed. These and other challenges lead Shephard et al. to conclude:

streaming existing videos is not for the faint-hearted; a wide range of issues will be encountered that must either be overcome or circumvented. Deciding which of these two responses best fits each problem is probably the toughest task and in each situation a rigorous cost-benefit analysis will be necessary.

In the Blake and Scanlon article, the stakeholders can be seen to be the different members of the evaluation team: CAL designers, course developers and educational technologists. But the use of video analysis to record detailed student behaviours introduces another stakeholder into the team - the student. Such an introduction is argued by Blake and Scanlon to enable a wider consideration of the potential benefits of CAL programmes.

In their article, Loewenberger and Bull report two examples in which key stakeholders influenced their survey. The first example is when staff from two faculties within the targeted institution resisted efforts to engage them in focus groups and interviews, which led to a change in methodology. The second example is that the majority of respondents in the final survey were reported to be those with high levels of seniority or experience. It is possible to suggest that more senior and experienced people returned the questionnaire because they had a bigger stake or interest in the relative costs and benefits of computerbased assessment. In both examples, stakeholders in the survey have exerted their power and influence in very different ways.

Nicol and Coen identify two key groups of stakeholders in their article: those tasked with costs analysis and those tasked with benefits analysis. Nicol and Coen note:

within institutions, those tasked with 'cost analysis normally have little expertise in benefits evaluation and those with benefits expertise have little costing experience, and these groups rarely work together.

The INSIGHT cost-benefit model described by Nicol and Coen can be seen as an attempt to draw the two sets of expertise together. Nicol and Coen conceptualize the model as a decision-support tool for senior managers in HE tasked with making investment decisions about initiatives in technology-supported teaching. They also suggest that a key stakeholder that will benefit from the application of such a model is the higher education sector, which will be able to evaluate the benefits of ICT from a wider institutional perspective. 
Finally, the discussion papers by Tompsett and Alsop and by Joyce and Lisewski challenge us to think about the learning technology community and the different views that different stakeholders within that community may hold. Joyce and Lisewski also suggest that some of these stakeholders represent 'powerful interest groups' from managerial, academic and political arenas.

Stakeholder diversity within the learning technology means that for those working within that community life is never dull. Perhaps we need to understand that diversity a little better in order to ensure that future developments can start from a springboard to success rather than a quagmire of 'nearly made it'.

\section{References}

DeRuyter, F. (1995), 'Evaluating outcomes in assistive technology: do we understand the commitment?', Assistive Technology, 7 (1), 3-16.

\section{Jane Seale}

Deputy Editor 